\title{
Wwox expression may predict benefit from adjuvant tamoxifen in randomized breast cancer patients
}

\author{
ANNA GÖTHLIN EREMO ${ }^{1}$, PIA WEGMAN ${ }^{1}$, OLLE STÅL $^{2}$, BO NORDENSKJÖLD ${ }^{2}$, \\ TOMMY FORNANDER ${ }^{3}$ and STEN WINGREN ${ }^{1}$
}

\author{
${ }^{1}$ School of Health and Medical Sciences, Örebro University, SE-70182 Örebro; ${ }^{2}$ Department of Clinical and \\ Experimental Medicine, Division of Oncology, Faculty of Health Sciences, Linköping University, SE-58185 Linköping; \\ ${ }^{3}$ Department of Oncology, Karolinska University Hospital, Stockholm South General Hospital, SE-11883 Stockholm, Sweden
}

Received November 15, 2012; Accepted January 4, 2013

DOI: $10.3892 /$ or.2013.2261

\begin{abstract}
Reduced or absent Wwox expression has recently been associated with tamoxifen resistance in breast cancer and has also been proposed as a candidate predictive marker for treatment. We aimed to investigate the correlation of Wwox expression with the outcome of tamoxifen treatment by examining tissues from 912 randomized breast cancer patients. Paraffin-embedded tissues from patient tumors were arranged on tissue microarray, and Wwox protein was stained using immunohistochemistry. After microscopic examination, the results were analyzed with Cox regression, Kaplan-Meier survival curves and the log-rank test. In the group of cases having a tumor absent for Wwox expression, there was no difference in recurrence-free survival between treated and untreated patients $(\mathrm{P}=0.81)$. For treated cases with a tumor expressing moderate or strong Wwox protein, recurrence-free survival was improved $(\mathrm{P}=0.001$ and $\mathrm{P}=0.003$, respectively). The test for interaction between Wwox and treatment response demonstrated a decreased risk of recurrence for treated patients with a moderate or strong Wwox expression ( $\mathrm{HR}=0.31,95 \% \mathrm{CI}$ 0.10-0.98 and HR=0.28, 95\% CI 0.08-0.97, respectively). Our results indicate that patients with high expression of Wwox may gain more benefit from treatment with tamoxifen.
\end{abstract}

\section{Introduction}

Patients with ER-positive tumors are candidates for endocrine therapy with the anti-estrogen tamoxifen. Unfortunately, up to $40 \%$ of ER-positive patients fail to respond, and a large fraction of initial responders develop resistance (1-5). Additional predictive markers for treatment selection are needed, given that resistant patients are at risk of disease relapse. One of these potential markers is Wwox, which is considered to be

Correspondence to: Anna Göthlin Eremo, Clinical Research Centre, Örebro University Hospital, SE-70185 Örebro, Sweden E-mail: anna.gothlin-eremo@oru.se

Key words: Wwox, breast cancer, tamoxifen, randomized patients a tumor suppressor and an inducer of apoptosis (6). Wwox includes two WW domains for protein-protein interactions and a short-chain alcohol dehydrogenase-reductase (SDR) domain that may play a part in sex steroid metabolism (6-8). The expression level of Wwox protein has shown to be significantly higher in ER-positive than in ER-negative tumors, suggesting a role in hormone signaling $(9,10)$. This is also supported by the fact that, in normal conditions, the highest expression of Wwox is observed in epithelial cells of hormonally regulated tissues such as ovaries, testes, prostate and mammary glands while thymus, adipose and connective tissues seem to lack Wwox (11-13). Furthermore, the Wwox gene is located on chromosome 16q23 and contains the fragile site FRA16D, the second most common fragile site in the human genome (14). Fragile sites are characterized by DNA instability and are frequently affected by genomic losses in several types of cancer. WWOX inactivation due to loss of heterozygosity ( $\mathrm{LOH})$ and hypermethylation has been reported, particularly in breast cancer (15-25). These events seem to appear early during carcinogenesis as they are found in ductal carcinoma in situ (26). Moreover, nearly all metastatic tissues show lower expression compared to their matched primary breast tumors, and therefore it is thought that loss of $W W O X$ expression plays an important role in the growth and progression of breast cancer $(21,27,28)$.

Intrinsic Wwox is reported mainly in mitochondria, however, nuclear translocation occurs in response to various stress signals and sex steroid hormones such as $17 \beta$-estradiol (E2). In breast cancer cells it seems that nuclear translocation occurs to a lesser extent as compared to prostate cancer cells, possibly due to interaction with cytoplasmic proteins (27). Through its WW domains, which are characterized by conserved tryptophan residues, Wwox binds to proline-rich motifs of several proteins in breast tissue. Wwox interaction seems to affect the function of other proteins, for example when bound to p53 it induces apoptosis in a synergistic manner $(29,30)$. AP $2 \gamma$, a transcription factor often upregulated in breast cancer, becomes sequestered in the cytoplasm by Wwox, preventing its entry into the nucleus $(31,32)$. Wwox binding to the intracellular domain (4ICD) of human epidermal growth factor receptor 4 (HER4, ErbB4) has been reported to generate similar cytoplasmic localization $(33,34)$. 
Depletion of WWOX in vitro was found to result in reduced ER expression and decreased sensitivity to tamoxifen (35). In a small non-randomized study correlating Wwox with tamoxifen resistance, it was shown that loss or reduced expression increased the probability of resistance more than 4-fold and that Wwox came out as a better predictor of response than PgR. (31). However, the full mechanism of de novo and acquired tamoxifen resistance is still largely unexplained; therefore, it is important to consider new potential markers. As Wwox has been proposed to predict response to tamoxifen treatment, we aimed to investigate the expression pattern of Wwox using a cohort of 912 randomized breast cancer patients. In the present study, we therefore compared Wwox protein expression with clinicopathological data of treated and untreated patients, which may bring us closer to elucidating the predictive value of Wwox.

\section{Materials and methods}

Patients. We analyzed tissues from low-risk breast cancer patients registered in a randomized tamoxifen trial, conducted in the Stockholm region between 1976 and 1990 (36). All patients $(n=1,780)$ were postmenopausal at the time of diagnosis, were required to have a tumor $\leq 30 \mathrm{~mm}$ in diameter and with no lymph node metastases (N0) (Table I). The patients were treated either with modified radical mastectomy or with breast-conserving surgery plus radiation therapy (50 Gy/5 weeks). Patients were randomized to tamoxifen therapy $(40 \mathrm{mg} / \mathrm{day})$ for 2 years $(\mathrm{n}=886)$ or no adjuvant endocrine treatment $(n=894)$. Tamoxifen treatment was initiated within 2-4 weeks after surgery and thus administered concurrently with radiation therapy. Patients without recurrence after 2 years were re-randomized to an additional 3 years of tamoxifen therapy, hence a total treatment period of 5 years, or no further treatment. Standard procedure for tissue collection was fixation in $4 \%$ phosphate-buffered formalin. The ER status was determined by isoelectric focusing with a cut-off level set to $0.05 \mathrm{fmol} / \mu \mathrm{g}$ DNA. The mean follow-up period was 17 years (36). The study was approved by the Ethics Committee of the Karolinska Institute. The ethical approval required no informed consent from the patients.

Breast tissue microarray. Archived breast tumor tissues from 912 of the 1,780 patients participating in the original study were collected. Representative formalin-fixed and paraffin-embedded tissue was chosen as a donor block for the tissue microarray (TMA). A section was cut from each block and stained with hematoxylin and eosin. Three morphologically representative regions from each section were chosen, and then cylindrical cores with a diameter of $0.8 \mathrm{~mm}$ were extracted and mounted in a recipient block. For each TMA, cores from liver tissue were mounted as internal controls. The TMA blocks were cut into $4-\mu \mathrm{m}$ sections using a microtome and mounted on glass slides. The TMA blocks were constructed using a manual arrayer (Beecher Instrument, Inc.).

Hormone receptor status. The Ventana BenchMark system with prediluted antibodies (anti-ER clone 6F11 and anti-PgR clone 16) was used to retrospectively determine the ER and progesterone (PgR) statuses of the 912 patients. Tumors with $>10 \%$ positively stained nuclei were considered positive. The immunohistochemical data regarding the ER status were used in this study; however, when data were missing, the results from the cytosol assay were used.

HER 2 analysis. Expression of HER 2 was analyzed by immunohistochemistry using the Dako AO0485 polyclonal rabbit antibody according to the manufacturer's instructions. The scoring was limited to the invasive tumor cells and graded 0 , $1+, 2+$ or $3+$. Patients having a score of $3+$ were considered HER2-positive.

Immunohistochemistry. Affinity isolated rabbit polyclonal antiWwox antibodies (cat. no W2143; Sigma-Aldrich, St. Louis, MO, USA) were used at a dilution of 1:300. Slides were deparaffinized in xylene and rehydrated in decreasing series of ethanol followed by Milli-Q water. Heat-induced antigen retrieval was performed in EDTA buffer $(1 \mathrm{mM}, \mathrm{pH}$ 8.0) for $1 \mathrm{~h}$ in a $99^{\circ} \mathrm{C}$ water container and cooled to room temperature (RT). Slides were blocked for endogenous peroxidase activity and unspecific binding using $3 \% \mathrm{H}_{2} \mathrm{O}_{2}$ for 10 min followed by $5 \%$ horse serum diluted in phosphate-buffered saline (PBS) for $1 \mathrm{~h}$ at RT. The slides were incubated with the primary antibody at $4^{\circ} \mathrm{C}$ for $16 \mathrm{~h}$, washed, and subsequent detection was carried out using the EnVision ${ }^{\mathrm{TM}}$ System (DakoCytomation, Glostrup, Denmark) with secondary horseradish peroxidase (HRP) polymer reagent for 20 min at RT and subsequently visualized with 3,3'diaminobenzidine (DAB). Sections were counterstained with hematoxylin and mounted. The positive and negative controls, prostate and thymus respectively, were stained appropriately. All washings in-between reactions were performed using PBS with $0.1 \%$ Tween (PBST).

Western blot analysis. To determine the specificity of the Wwox antibody, western blot analysis was performed. Protein $(1 \mathrm{mg} / \mathrm{ml})$ from lysed MCF-7 cells was separated by $12 \%$ SDS-PAGE and transferred to polyvinylidene difluoride membranes (Bio-Rad Laboratories, Hercules, CA, USA) for $1 \mathrm{~h}$ at $100 \mathrm{~V}$. MCF-7 culturing and protein extraction were performed according to the standard protocol. The membranes were blocked for unspecific binding for $1 \mathrm{~h}$ at RT using PBST with 5\% horse serum and then incubated with primary antibody against Wwox $(1: 3,000)$ at $4^{\circ} \mathrm{C}$ for $16 \mathrm{~h}$. For detection, the membranes were incubated with a HRP-conjugated secondary goat anti-rabbit antibody 1:50,000 (Santa Cruz Biotechnology, Santa Cruz, CA, USA) for $1 \mathrm{~h}$ at RT and visualized using the ECL Advance Western Blotting Detection kit (GE Healthcare, Buckinghamshire, UK) according to the manufacturer's instructions.

TMA evaluation. The TMA slides were examined with an Olympus BX41 light microscope (Olympus Life Science Europe GMBH, Hamburg, Germany) connected to a Leica DFC420 digital microscope camera (Leica Microsystems, Heerbrugg, Switzerland). Two investigators (A.G.E. and S.W.) evaluated the slides independently and were blinded to any clinical data or patient outcome. The cut-off level was set at $>10 \%$ of positively stained tumor cells. The intensity of cytoplasmic and nuclear Wwox staining was graded as 
Table I. Comparison of the distribution of patients in regards to tumor and treatment characteristics.

\begin{tabular}{|c|c|c|c|}
\hline & \multicolumn{3}{|c|}{ No. of patients (\%) } \\
\hline & $\begin{array}{l}\text { Patients in the } \\
\text { present study }(n=912)\end{array}$ & $\begin{array}{c}\text { Patients with } \\
\text { Wwox expression }(n=686)\end{array}$ & $\begin{array}{l}\text { Original randomized } \\
\text { study }(\mathrm{n}=1,780)\end{array}$ \\
\hline \multicolumn{4}{|c|}{ Estrogen receptor } \\
\hline Positive & $684(77)$ & $519(77)$ & $1,183(80)$ \\
\hline Negative & $200(23)$ & $153(23)$ & $296(20)$ \\
\hline Unavailable & 28 & 14 & 301 \\
\hline \multicolumn{4}{|c|}{ Progesterone receptor } \\
\hline Positive & $379(48)$ & $299(47)$ & $590(48)$ \\
\hline Negative & $416(52)$ & $333(53)$ & $627(52)$ \\
\hline Unavailable & 117 & 54 & 563 \\
\hline \multicolumn{4}{|c|}{ Tumor diameter (mm) } \\
\hline$\leq 20$ & $697(79)$ & $515(76)$ & $1,393(81)$ \\
\hline$>20$ & $189(21)$ & $159(24)$ & $323(19)$ \\
\hline Unavailable & 26 & 12 & 64 \\
\hline \multicolumn{4}{|c|}{ Tamoxifen treatment } \\
\hline Yes & $473(52)$ & $348(51)$ & $886(50)$ \\
\hline No & $439(48)$ & $338(49)$ & $894(50)$ \\
\hline
\end{tabular}

follows; 0 , negative; 1 , weak/moderate or 2 , strong. A new category of total Wwox expression was calculated by adding the scores together: 0 , negative; $1-2$, weak/moderate; and 3-4, strong. Cases that were considered positive for Wwox expression had to have at least an intensity of 1 in the cytoplasm, in the nucleus or in both. In the case of non-consistent scoring between investigators, a consensus score was established after re-evaluation.

Statistical analysis. To examine the relationship between the different levels of protein expression and tumor characteristics as well as the correlation between nuclear and cytoplasmic expression, we used Pearson's Chi-square tests. The time for recurrence-free survival was calculated as the time between diagnosis and local recurrence of breast cancer, distant metastasis or death from breast cancer. The recurrence-free survival rates and the difference in survival rate between treatment groups were estimated using the Kaplan-Meier method and the log-rank test, respectively. The interaction between Wwox and treatment was calculated with a univariate Cox regression model. In addition, hazard ratios (relative hazard, HR) with a $95 \%$ confidence interval $(95 \% \mathrm{CI})$ were assessed using a multivariate Cox regression model in order to adjust for the tumor characteristics between the different expression profiles. All P-values $<0.05$ were considered to indicate a statistically significant result. The Statistical Package for the Social Sciences (SPSS), version 15.0, was used to perform all statistical analyses.

\section{Results}

Protein expression of Wwox. Protein expression of Wwox was assessed using immunohistochemical staining performed on TMA slides, comprising tumor tissues from 912 breast cancer patients. Wwox scoring was accessible in 686 cases (75.2\%), and the patient distribution of characteristics in comparison to the original cohort is described in Table I. Between cases available on TMA and those scored for Wwox expression, there were no statistical differences regarding the distribution of characteristics. From the accessible cases, expression of cytoplasmic Wwox was observed in 560 (81.6\%) cases and expression of nuclear Wwox was observed in $564(82.2 \%)$ cases (Table II). There were $62(9.0 \%)$ cases who were positive for nuclear but not cytoplasmic Wwox and 58 (8.5\%) who were positive for cytoplasmic but not nuclear expression of Wwox. However, 502 (73.2\%) cases had Wwox expression in both locations $(\mathrm{OR}=8.9,95 \% \mathrm{CI}$ 5.7-13.9, $\mathrm{P}<0.0001)$. Different patterns of immunohistochemical staining along with the result from the positive and negative controls are shown in Fig. 1. The specificity of the antibody was verified with western blot analysis (data not shown).

Protein expression of Wwox in correlation with prognostic factors. The analysis of Wwox and its relationship with tumor characteristics demonstrated a correlation between the grade of nuclear Wwox expression and positive ER and PgR status $(\mathrm{P}=0.0001$ and $\mathrm{P}=0.045$, respectively, Table II) and the grade of cytoplasmic Wwox was correlated to the HER2 status $(\mathrm{P}=0.001)$. Several HER2-positive patients were also ER-positive (32 of 76) but the Chi-square test for trend showed no correlation between Wwox expression and ER status in these patients $(\mathrm{P}=0.24)$. There were no significant correlations to other characteristics.

Wwox prognostic relevance in breast cancer. Among the 348 patients treated with tamoxifen, 271 (77.9\%) had an ER-positive tumor and 5 (1.4\%) had an unknown ER status. Among the 338 patients who did not received endocrine therapy, 248 (73.4\%) 
Table II. Statistical correlations between Wwox expression and tumor characteristics in the 686 breast cancer patients included in the study.

No. of patients (\% within each group)

\begin{tabular}{|c|c|c|c|c|c|c|c|c|}
\hline & \multicolumn{3}{|c|}{ Nuclear Wwox expression } & \multirow[b]{2}{*}{ P-value ${ }^{a / b}$} & \multicolumn{3}{|c|}{ Cytoplasmic Wwox expression } & \multirow[b]{2}{*}{ P-value ${ }^{a / b}$} \\
\hline & Negative & Moderate & Strong & & Negative & Moderate & Strong & \\
\hline Total & $122(17.8)$ & $366(53.4)$ & $198(28.9)$ & & $126(18.4)$ & $393(57.3)$ & $167(24.3)$ & \\
\hline \multicolumn{9}{|c|}{ Estrogen receptor } \\
\hline Positive & $83(69.2)$ & $269(74.9)$ & $167(86.5)$ & & 97 (78.9) & $295(76.4)$ & 127 (77.9) & \\
\hline Negative & $37(30.8)$ & $90(25.1)$ & $26(13.5)$ & $0.001 / 0.0001$ & $26(21.1)$ & $91(23.6)$ & $36(22.1)$ & $0.830 / 0.899$ \\
\hline \multicolumn{9}{|c|}{ Progesterone receptor } \\
\hline Positive & $41(39.4)$ & $161(47.2)$ & 97 (51.9) & & $53(47.3)$ & $178(49.7)$ & $68(42.0)$ & \\
\hline Negative & $63(60.6)$ & $180(52.8)$ & $90(48.1)$ & $0.125 / 0.045$ & $59(52.7)$ & $180(50.3)$ & $94(58.0)$ & $0.261 / 0.292$ \\
\hline \multicolumn{9}{|c|}{ Tumor diameter (mm) } \\
\hline$\leq 20$ & $93(78.2)$ & $263(73.1)$ & $159(81.5)$ & & $100(80.0)$ & $284(74.3)$ & $131(78.4)$ & \\
\hline$>20$ & $26(21.8)$ & $97(26.9)$ & $36(18.5)$ & $0.071 / 0.286$ & $25(20.0)$ & $98(25.7)$ & $36(21.6)$ & $0.336 / 0.880$ \\
\hline \multicolumn{9}{|c|}{ Tamoxifen treatment } \\
\hline Yes & $54(44.3)$ & $192(52.5)$ & $102(51.5)$ & & $57(45.2)$ & $203(51.7)$ & $88(52.7)$ & \\
\hline No & $68(55.7)$ & $174(47.5)$ & $96(48.5)$ & $0.282 / 0.285$ & $69(54.8)$ & $190(48.3)$ & $79(47.3)$ & $0.384 / 0.232$ \\
\hline \multicolumn{9}{|l|}{ HER2 } \\
\hline Positive & $12(10.5)$ & $44(12.8)$ & $20(10.8)$ & & $5(4.3)$ & 44 (11.9) & $27(17.1)$ & \\
\hline Negative & $102(89.5)$ & $300(87.2)$ & $166(89.2)$ & $0.705 / 0.928$ & $111(95.7)$ & $326(88.1)$ & $131(82.9)$ & $0.005 / 0.001$ \\
\hline
\end{tabular}

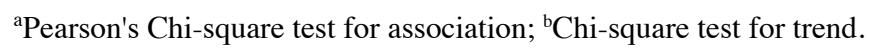
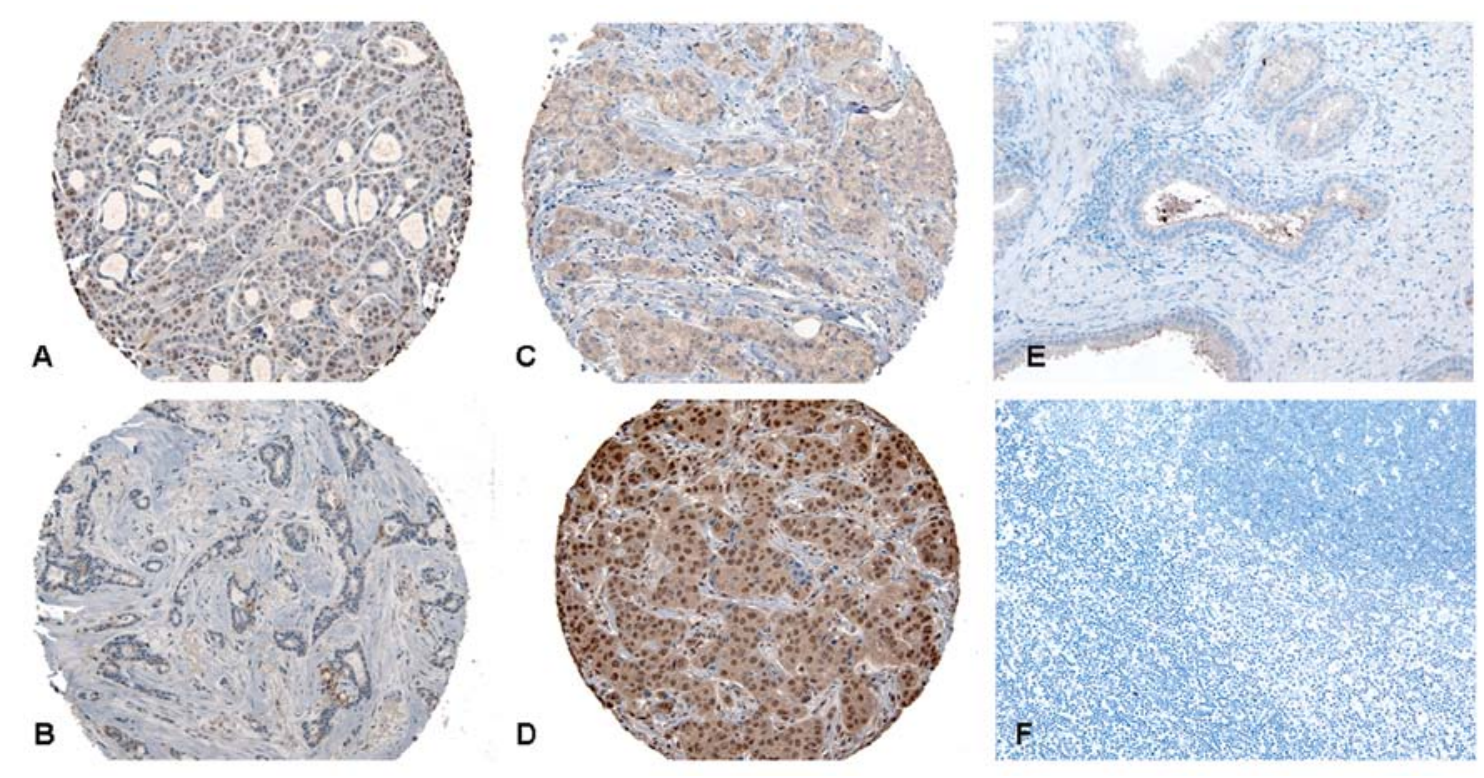

Figure 1. Micrographs showing the different patterns of Wwox immunohistochemical staining. (A) Moderate Wwox expression in nuclei surrounded by negative cytoplasmic expression. (B) Tumor cells lacking Wwox expression. (C) Negative nuclei with a moderate cytoplasmic Wwox expression. (D) Strong nuclear and cytoplasmic Wwox expression in all tumor cells. (A-D) Selected tissues from the included patients at x10 magnification. (E) Prostate tissue with moderate protein expression of Wwox. (F) Tissue from thymus showing absence of Wwox expression.

were ER-positive and 9 (2.7\%) had an unknown ER status. To uncover any prognostic relevance of Wwox in breast cancer, the group of patients without endocrine treatment was selected for analysis. These results showed no difference in recurrencefree survival between patients with or without cytoplasmic Wwox $(\mathrm{P}=0.98)$, nuclear Wwox $(\mathrm{P}=0.74)$ or total Wwox 

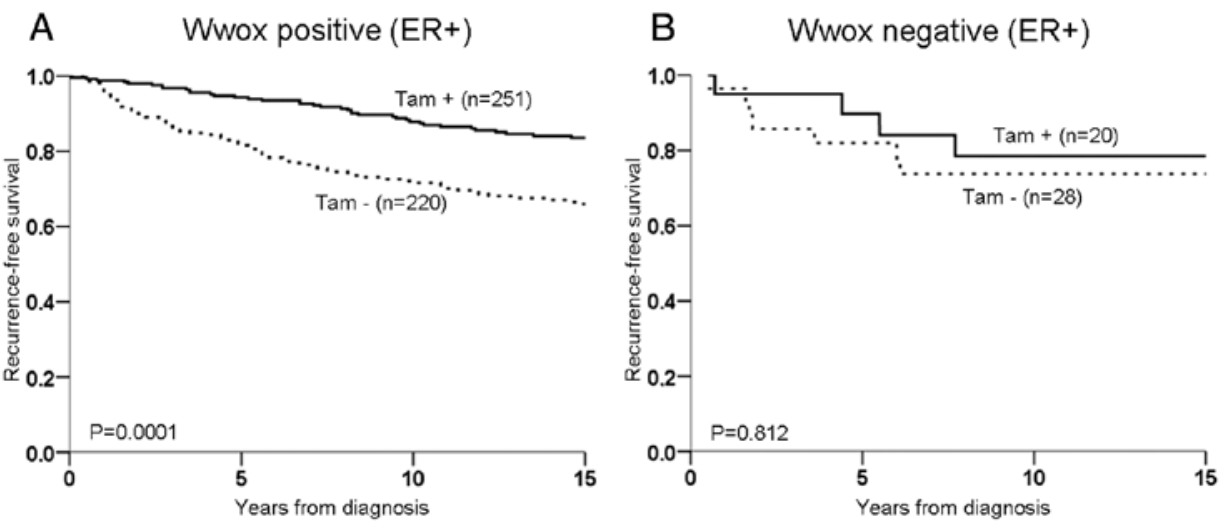

Figure 2. Kaplan-Meier curves representing the cumulative recurrence-free survival in ER-positive patients (ER ${ }^{+}$) with a Wwox-positive (A) or Wwoxnegative (B) breast tumor.
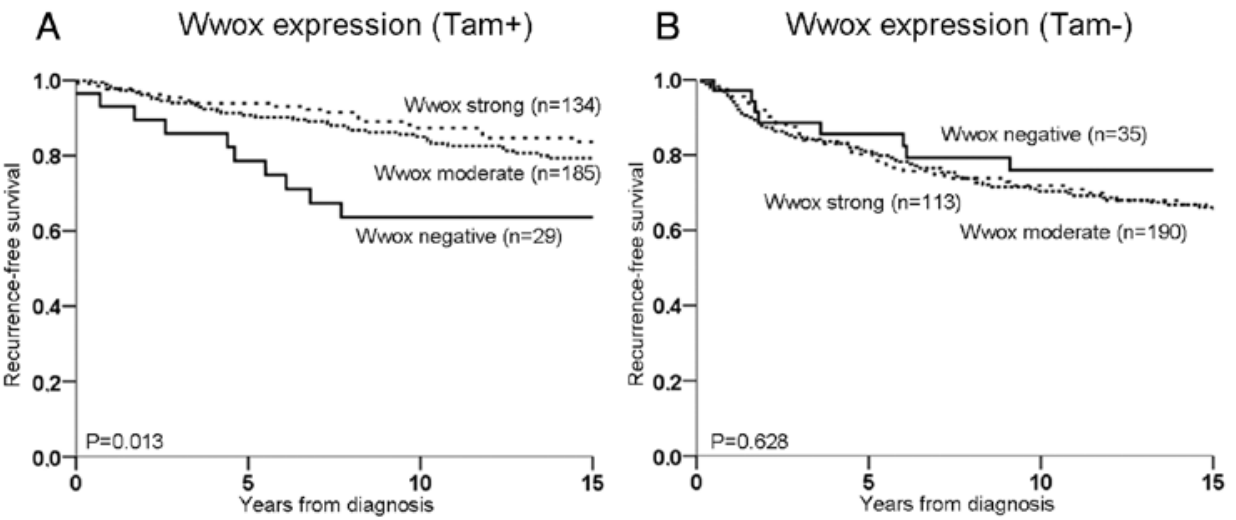

Figure 3. Kaplan-Meier curves representing the cumulative recurrence-free survival in the tamoxifen-treated patients that were scored for Wwox expression. (A) Tamoxifen-treated patients with moderate or strong tumor expression of Wwox showed improved outcome compared to patients with no expression. (B) For untreated patients, there was no significant difference in survival dependent on Wwox expression.

( $\mathrm{P}=0.38)$. Subanalysis of the few cases with single nuclear or single cytoplasmic expression did not show any difference in recurrence-free survival $(\mathrm{P}=0.69)$. When selecting only ER-positive patients, there was still no difference in survival observed in regards to Wwox staining $(\mathrm{P}=0.74, \mathrm{P}=0.26$ and $\mathrm{P}=0.69$, respectively). In the multivariate analysis including ER, PgR and tumor size, moderate or strong Wwox expression were not independent predictive markers $(\mathrm{P}=0.83$ and $\mathrm{P}=0.93$, respectively).

Wwox expression and response to tamoxifen treatment. When tamoxifen was introduced into the statistical model, the ER-positive patients were selected for further analyses, as this group of patients currently receive tamoxifen therapy. The patients were divided in regards to Wwox tumor expression, and tamoxifen treatment was found to result in an improved recurrence-free survival for those expressing Wwox $(\mathrm{P}=0.0001$; moderate and strong expression $\mathrm{P}=0.001$ and $\mathrm{P}=0.003$, respectively, Fig. 2A). However, in patients lacking Wwox expression no difference in recurrence-free survival was noted regardless of treatment $(\mathrm{P}=0.812$, Fig. $2 \mathrm{~B})$. Irrespective of ER, tamoxifen-treated patients with a moderate or strong Wwox expression had an improved recurrence-free survival compared to patients without Wwox expression $(\mathrm{P}=0.013$, Fig. 3A). When moderate and strong expression were grouped together and termed Wwox-positive, the difference was even greater $(\mathrm{P}=0.005)$. By contrast, no difference in recurrencefree survival was noted among untreated patients according to different levels of Wwox expression ( $\mathrm{P}=0.63$, Fig. 3B). The test for the interaction between Wwox and tamoxifen treatment demonstrated a decreased risk of recurrence for treated patients with a moderate or strong Wwox expression $(\mathrm{HR}=0.31,95 \% \mathrm{CI} 0.10-0.98$ and $\mathrm{HR}=0.28,95 \% \mathrm{CI} 0.08-0.97$, respectively).

\section{Discussion}

The Wwox protein is a relatively newly discovered tumor suppressor with a complex biological behavior that is still under investigation. Wwox has been shown to interact with a growing list of proteins and seems to be involved in numerous cellular pathways. High expression of Wwox has been correlated with a more favorable survival of breast cancer patients in several studies $(10,12,26,37)$. As Guler et al (31) demonstrated an association between Wwox protein expression and tamoxifen resistance, we decided to further investigate the potential of 
Wwox to predict tamoxifen response. In the present study, we evaluated Wwox protein expression in tumors from 686 randomized breast cancer patients and correlated the results to clinicopathological data as well as to tamoxifen treatment. Breast cancer patients treated with tamoxifen had a significantly prolonged recurrence-free survival when their tumor expressed higher levels of the Wwox protein. We also found a significant difference in recurrence-free survival between Wwox-positive and -negative cases among the patients treated with tamoxifen but not the untreated patients. The test of interaction using Cox's regression demonstrated that the risk of recurrence decreased in tamoxifen-treated patients when their tumor expressed the Wwox protein. This is in line with Guler's cohort where the probability of tamoxifen resistance was increased more than 4-fold when Wwox protein expression was reduced. According to Guler et al, the predictive value of Wwox was better than PgR (31); however, multivariate analysis of our results did not reveal such a scenario. Furthermore, our results did not support that Wwox has any prognostic relevance but our findings may indicate that Wwox is involved in pathways interfering with tamoxifen response. There are several known mechanisms leading to tamoxifen resistance, such as drug metabolism, changes in co-regulatory proteins, adaption and tolerance, but to date, there are difficulties in predicting the outcome of treatment based on these factors (1). In addition, ER and sex hormone steroids can directly interact with cytoplasmic signaling molecules such as the tyrosine kinases Src and PI3K, initiate extranuclear pathways leading to gene expression $(38,39)$. This crosstalk between signaling pathways and ER further complicates the understanding of resistance mechanisms. It is unclear how Wwox contributes to tamoxifen response given that its normal functions are yet to be fully explored. Wwox appears mainly to be involved in protein-protein interactions in the cytoplasm, yet there are indications of nuclear translocation. This event has been shown in COS7 fibroblasts after exposure to E2, which suggests a hormonally influenced nuclear function $(27,30)$. Watanabe et al (40) carried out an immunohistochemical analysis of Wwox and observed nuclear staining in mammary epithelia and in cell lines during confluent culture conditions. Conversely, when Nunez et al (13) performed a survey on the topographic distribution of Wwox protein expression they found only cytoplasmic staining. Importantly, this survey was carried out in normal, not cancerous, human tissues. As immunohistochemical studies are subject to the specificity of the antibodies, we used the survey constructed by Nunez et al to preference control tissues for validation of our selected antibody (Fig. 1). Moreover, western blot analysis identified one single band of predicted size also suggesting specificity. We found that most of our cases had a combination of nuclear and cytoplasmic expression of Wwox and that the localization of Wwox did not seem to influence recurrence-free survival of breast cancer patients. Nevertheless, there was a correlation between nuclear Wwox and ER and a correlation between cytoplasmic Wwox and HER2. As ER is a nuclear receptor and HER2 is a membrane-bound receptor, the correlations noted may reflect a relationship between cellular positions more than a functional association. Even so, the correlations may also indicate a possible Wwox involvement in both ER and HER2 signaling as the pathways intersect $(39,41)$. The highest normal expression of Wwox is found in hormonally influenced tissues including the prostate, and therefore it has been suggested to play a part in prostate cancer $(25,41)$. In the event of prostate cells transforming to a cancerous and metastatic state, Wwox is phosphorylated and translocate to the nucleus (27).

First, the interactive ability of Wwox is modified via phosphorylation at Tyr33 by Src, which is induced by stress stimuli and sex steroid hormones such as estradiol (29). Src is frequently upregulated in cancer and is also known to interact with HER2, promoting growth and survival of breast cancer cells (42). It has also been implied that higher levels of cytoplasmic Src are associated with poor response to tamoxifen therapy (43). In a normal state, an elevated Src expression could lead to Wwox activation resulting in Wwox-mediated tumor suppression. Thus, the loss of Wwox may therefore increase the oncogenic effect of $\mathrm{Src}$ which may result in a worse outcome of cancer.

Secondly, Wwox expression has also been shown to correlate with activator protein $2 \gamma(\mathrm{AP} 2 \gamma)$, a transcription factor often upregulated in breast tumors. Reduced Wwox protein expression enhances the AP $2 \gamma$ transcriptional effect in the nucleus, as AP $2 \gamma$ is retained to a lesser extent in the cytoplasm without Wwox interaction $(31,32)$. AP $2 \gamma$ regulates HER2 expression and other genes involved in tumor growth, and loss of Wwox could theoretically be biologically associated with increased HER2 expression (42-44). Qin et al (41) reported that ectopically expressed Wwox reduced HER2 expression via AP2 $\gamma$ interaction in prostate cancer cells, although it required signaling through the androgen receptor. The HER2positive cases in the present study appeared to be associated with higher Wwox expression, which is in contrast to the report by Qin et al (41). This might be explained by the fact that our cohort included low-risk patients with a fewer number of HER2-positive cases.

A third binding partner to Wwox is HER4, which is another member of the epidermal growth factor receptor family. HER4 and Wwox interact through the intracellular domain (4ICD) of HER4. Keeping 4ICD in the cytoplasm by Wwox promotes apoptosis as 4ICD harbors a pro-apoptotic BH3-domain. 4ICD located in the nucleus instead acts as a co-activator to ER and contributes to growth and proliferation $(33,34,45,46)$. Co-expression of HER4 and Wwox is associated with a favorable outcome in breast cancer compared to when HER4 is expressed in the absence of Wwox (34). As one of its drug effects, tamoxifen obstructs the association between ER and its co-activators by changing ER conformation (4). It is suggested that HER4 might be important in drug resistance in breast cancer, as tamoxifen reduces the possibility of 4ICD binding to the ER complex. When Wwox expression is lost or reduced, more 4ICD is available in the nucleus for promoting cell survival. Taken together, it is plausible that loss of Wwox expression may affect the response of endocrine therapy in breast cancer. As tamoxifen is one of the most commonly used cancer drugs and breast cancer affects one in ten women in the Western world, there is a considerable need for reliable predictive markers for treatment selection. Since the present cohort was built up by historically exclusive material placed on TMA, there are obvious limitations to the use of the methods. Nevertheless, we were able to evaluate the influence of Wwox on tamoxifen therapy in a large number of randomized 
patients using immunohistochemistry. Collectively, we found that Wwox expression corresponds to improved recurrencefree survival even though our results need further validation in other trials. In conclusion, the present study emphasizes the existing theory of Wwox as a possible predictor of tamoxifen response.

\section{Acknowledgements}

This study was supported by grants from the Percy Falk Foundation for Research in Breast and Prostate Cancer, Stockholm, Sweden.

\section{References}

1. Ring A and Dowsett M: Mechanisms of tamoxifen resistance. Endocr Relat Cancer 11: 643-658, 2004.

2. Greenspan EM: Tamoxifen in early breast cancer. Lancet 1: 655, 1983.

3. Rose C, Thorpe SM, Andersen KW, Pedersen BV, Mouridsen HT, Blichert-Toft $\mathrm{M}$ and Rasmussen BB: Beneficial effect of adjuvant tamoxifen therapy in primary breast cancer patients with high oestrogen receptor values. Lancet 1: 16-19, 1985.

4. Shiau AK, Barstad D, Loria PM, Cheng L, Kushner PJ, Agard DA and Greene GL: The structural basis of estrogen receptor/coactivator recognition and the antagonism of this interaction by tamoxifen. Cell 95: 927-937, 1998.

5. Stewart HJ and Prescott R: Adjuvant tamoxifen therapy and receptor levels. Lancet 1: 573, 1985.

6. Paige AJ, Taylor KJ, Taylor C, Hillier SG, Farrington S, Scott D, Porteous DJ, Smyth JF, Gabra H and Watson JE: WWOX: a candidate tumor suppressor gene involved in multiple tumor types. Proc Natl Acad Sci USA 98: 11417-11422, 2001.

7. Aqeilan RI, Trapasso F, Hussain S, Costinean S, Marshall D, Pekarsky Y, Hagan JP, Zanesi N, Kaou M, Stein GS, et al Targeted deletion of Wwox reveals a tumor suppressor function. Proc Natl Acad Sci USA 104: 3949-3954, 2007.

8. Bednarek AK, Laflin KJ, Daniel RL, Liao Q, Hawkins KA and Aldaz CM: WWOX, a novel WW domain-containing protein mapping to human chromosome 16q23.3-24.1, a region frequently affected in breast cancer. Cancer Res 60: 2140-2145, 2000.

9. Nunez MI, Ludes-Meyers J, Abba MC, Kil H, Abbey NW, Page RE, Sahin A, Klein-Szanto AJ and Aldaz CM: Frequent loss of WWOX expression in breast cancer: correlation with estrogen receptor status. Breast Cancer Res Treat 89: 99-105, 2005.

10. Płuciennik E, Kusińska R, Potemski P, Kubiak R, Kordek R and Bednarek AK: WWOX - the FRA16D cancer gene: expression correlation with breast cancer progression and prognosis. Eur J Surg Oncol 32: 153-157, 2006.

11. Driouch K, Prydz H, Monese R, Johansen H, Lidereau R and Frengen E: Alternative transcripts of the candidate tumor suppressor gene, WWOX, are expressed at high levels in human breast tumors. Oncogene 21: 1832-1840, 2002.

12. Guler G, Uner A, Guler N, Han SY, Iliopoulos D, Hauck WW, McCue P and Huebner K: The fragile genes FHIT and WWOX are inactivated coordinately in invasive breast carcinoma. Cancer 100: 1605-1614, 2004.

13. Nunez MI, Ludes-Meyers $\mathbf{J}$ and Aldaz CM: WWOX protein expression in normal human tissues. J Mol Histol 37: 115-125, 2006.

14. Bednarek AK, Keck-Waggoner CL, Daniel RL, Laflin KJ, Bergsagel PL, Kiguchi K, Brenner AJ and Aldaz CM: WWOX, the FRA16D gene, behaves as a suppressor of tumor growth. Cancer Res 61: 8068-8073, 2001.

15. Aqeilan RI, Kuroki T, Pekarsky Y, Albagha O, Trapasso F, Baffa R, Huebner K, Edmonds P and Croce CM: Loss of WWOX expression in gastric carcinoma. Clin Cancer Res 10: 3053-3058, 2004.

16. Baykara O, Demirkaya A, Kaynak K, Tanju S, Toker A and Buyru N: WWOX gene may contribute to progression of nonsmall-cell lung cancer (NSCLC). Tumour Biol 31: 315-320, 2010.
17. Bloomston M, Kneile J, Butterfield M, Dillhoff M, Muscarella P, Ellison EC, Melvin WS, Croce CM, Pichiorri F, Huebner, et al: Coordinate loss of fragile gene expression in pancreatobiliary cancers: correlations among markers and clinical features. Ann Surg Oncol 16: 2331-2338, 2009.

18. Fabbri M, Iliopoulos D, Trapasso F, Aqeilan RI, Cimmino A, Zanesi N, Yendamuri S, Han SY, Amadori D, Huebner K, et al: WWOX gene restoration prevents lung cancer growth in vitro and in vivo. Proc Natl Acad Sci USA 102: 15611-15616, 2005.

19. Finnis M, Dayan S, Hobson L, Chenevix-Trench G, Friend K, Ried K, Venter D, Woollatt E, Baker E and Richards RI: Common chromosomal fragile site FRA16D mutation in cancer cells. Hum Mol Genet 14: 1341-1349, 2005.

20. Iliopoulos D, Guler G, Han SY, Johnston D, Druck T, McCorkell KA, Palazzo J, McCue PA, Baffa R and Huebner K: Fragile genes as biomarkers: epigenetic control of WWOX and FHIT in lung, breast and bladder cancer. Oncogene 24: 1625-1633, 2005.

21. Iliopoulos D, Fabbri M, Druck T, Qin HR, Han SY and Huebner K: Inhibition of breast cancer cell growth in vitro and in vivo: effect of restoration of Wwox expression. Clin Cancer Res 13: 268-274, 2007

22. Kuroki T, Yendamuri S, Trapasso F, Matsuyama A, Aqeilan RI, Alder H, Rattan S, Cesari R, Nolli ML, Williams NN, et al: The tumor suppressor gene WWOX at FRA16D is involved in pancreatic carcinogenesis. Clin Cancer Res 10: 2459-2465, 2004.

23. O'Keefe LV, Liu Y, Perkins A, Dayan S, Saint R and Richards RI: FRA16D common chromosomal fragile site oxido-reductase (FOR/WWOX) protects against the effects of ionizing radiation in Drosophila. Oncogene 24: 6590-6596, 2005.

24. Park SW, Ludes-Meyers J, Zimonjic DB, Durkin ME, Popescu NC and Aldaz CM: Frequent downregulation and loss of WWOX gene expression in human hepatocellular carcinoma. Br J Cancer 91: 753-759, 2004.

25. Qin HR, Iliopoulos D, Semba S, Fabbri M, Druck T, Volinia S, Croce CM, Morrison CD, Klein RD and Huebner K: A role for the WWOX gene in prostate cancer. Cancer Res 66: 6477-6481, 2006.

26. Guler G, Uner A, Guler N, Han SY, Iliopoulos D, McCue P and Huebner K: Concordant loss of fragile gene expression early in breast cancer development. Pathol Int 55: 471-478, 2005.

27. Chang NS, Schultz L, Hsu LJ, Lewis J, Su M and Sze CI: 17beta-Estradiol upregulates and activates WOX1/WWOXv1 and WOX2/WWOXv2 in vitro: potential role in cancerous progression of breast and prostate to a premetastatic state in vivo. Oncogene 24: 714-723, 2005.

28. Guler G, Himmetoglu C, Jimenez RE, Geyer SM, Wang WP, Costinean S, Pilarski RT, Morrison C, Suren D, Liu J, et al: Aberrant expression of DNA damage response proteins is associated with breast cancer subtype and clinical features. Breast Cancer Res Treat 129: 421-432, 2010.

29. Aqeilan RI, Pekarsky Y, Herrero JJ, Palamarchuk A, Letofsky J, Druck T, Trapasso F, Han SY, Melino G, Huebner K, et al: Functional association between Wwox tumor suppressor protein and p73, a p53 homolog. Proc Natl Acad Sci USA 101: 4401-4406, 2004.

30. Chang NS, Doherty J, Ensign A, Schultz L, Hsu LJ and Hong Q: WOX1 is essential for tumor necrosis factor-, UV light-, staurosporine-, and p53-mediated cell death, and its tyrosine 33-phosphorylated form binds and stabilizes serine 46-phosphorylated p53. J Biol Chem 280: 43100-43108, 2005.

31. Guler G, Iliopoulos D, Guler N, Himmetoglu C, Hayran M and Huebner K: Wwox and Ap2gamma expression levels predict tamoxifen response. Clin Cancer Res 13: 6115-6121, 2007.

32. Guler G, Huebner K, Himmetoglu C, Jimenez RE, Costinean S, Volinia S, Pilarski RT, Hayran M and Shapiro CL: Fragile histidine triad protein, WW domain-containing oxidoreductase protein Wwox, and activator protein 2gamma expression levels correlate with basal phenotype in breast cancer. Cancer 115: 899-908, 2009.

33. Aqeilan RI, Donati V, Palamarchuk A, Trapasso F, Kaou M, Pekarsky Y, Sudol M and Croce CM: WW domain-containing proteins, WWOX and YAP, compete for interaction with ErbB-4 and modulate its transcriptional function. Cancer Res 65: 6764-6772, 2005.

34. Aqeilan RI, Donati V, Gaudio E, Nicoloso MS, Sundvall M, Korhonen A, Lundin J, Isola J, Sudol M, Joensuu H, et al: Association of Wwox with ErbB4 in breast cancer. Cancer Res 67: 9330-9336, 2007. 
35. Abdeen SK, Salah Z, Maly B, Smith Y, Tufail R, Abu-Odeh M, Zanesi N, Croce CM, Nawaz Z and Aqeilan RI: Wwox inactivation enhances mammary tumorigenesis. Oncogene 30 3900-3906, 2011.

36. Rutqvist LE, Johansson H and Group SBCS: Long-term follow-up of the randomized Stockholm trial on adjuvant tamoxifen among postmenopausal patients with early stage breast cancer. Acta Oncol 46: 133-145, 2007

37. Wang X, Chao L, Ma G, Chen L, Zang Y and Sun J: The prognostic significance of WWOX expression in patients with breast cancer and its association with the basal-like phenotype. J Cancer Res Clin Oncol 137: 271-278, 2011.

38. Fox EM, Andrade J and Shupnik MA: Novel actions of estrogen to promote proliferation: integration of cytoplasmic and nuclear pathways. Steroids 74: 622-627, 2009.

39. Yang Z, Barnes CJ and Kumar R: Human epidermal growth factor receptor 2 status modulates subcellular localization of and interaction with estrogen receptor alpha in breast cancer cells. Clin Cancer Res 10: 3621-3628, 2004

40. Watanabe A, Hippo Y, Taniguchi H, Iwanari H, Yashiro M, Hirakawa K, Kodama T and Aburatani H: An opposing view on WWOX protein function as a tumor suppressor. Cancer Res 63: 8629-8633, 2003.

41. Qin HR, Iliopoulos D, Nakamura T, Costinean S, Volinia S, Druck T, Sun J, Okumura H and Huebner K: Wwox suppresses prostate cancer cell growth through modulation of ErbB2mediated androgen receptor signaling. Mol Cancer Res 5: 957-965, 2007.
42. Belsches-Jablonski AP, Biscardi JS, Peavy DR, Tice DA, Romney DA and Parsons SJ: Src family kinases and HER2 interactions in human breast cancer cell growth and survival. Oncogene 20: 1465-1475, 2001.

43. Morgan L, Gee J, Pumford S, Farrow L, Finlay P, Robertson J, Ellis I, Kawakatsu H, Nicholson R and Hiscox S: Elevated Src kinase activity attenuates Tamoxifen response in vitro and is associated with poor prognosis clinically. Cancer Biol Ther 8: $1550-1558,2009$

44. Pellikainen J, Naukkarinen A, Ropponen K, Rummukainen J, Kataja V, Kellokoski J, Eskelinen M and Kosma VM: Expression of HER 2 and its association with AP-2 in breast cancer. Eur J Cancer 40: 1485-1495, 2004.

45. Junttila TT, Sundvall M, Lundin M, Lundin J, Tanner M, Härkönen P, Joensuu H, Isola J and Elenius K: Cleavable ErbB4 isoform in estrogen receptor-regulated growth of breast cancer cells. Cancer Res 65: 1384-1393, 2005.

46. Naresh A, Thor AD, Edgerton SM, Torkko KC, Kumar R and Jones FE: The HER4/4ICD estrogen receptor coactivator and $\mathrm{BH} 3$-only protein is an effector of tamoxifen-induced apoptosis. Cancer Res 68: 6387-6395, 2008. 